\title{
Erratum to: 1st Joint ANIRCEF-SISC Congress (meeting abstracts)
}

\author{
Paolo Martelletti
}

\section{Erratum}

Following publication of this supplement [1] it was brought to our attention that the affiliations of Dr Francesco Pierelli and Dr Ferdinando Nicoletti were incorrectly listed in a number of the abstracts published as part of this supplement [2-15].

Please note that for twelve abstracts listed [2-13] Dr Francesco Pierelli's affiliation should be as follows:

1. Department of Medico-Surgical Sciences and Biotechnologies, Sapienza University of Rome, Rome, Italy

2. I.R.C.C.S. Neuromed, Pozzilli, Italy

Further to this, for two abstracts listed [13, 14] Dr Ferdinando Nicoletti's affiliation should be as follows:

1. Department of Physiology and Pharmacology, University Sapienza of Rome, Rome, Italy

2. I.R.C.C.S. Neuromed, Pozzilli, Italy

Received: 7 December 2016 Accepted: 7 December 2016

Published online: 31 January 2017

\section{References}

1. The Authors. 1st Joint ANIRCEF-SISC Congress. The Journal of Headache and Pain 2015, 16(Suppl 1)

2. Di Lenola D et al (2015) 0024. Transcutaneous supraorbital nerve stimulation enhances somatosensory thalamic activity in migraine between attacks: a central mechanism of clinical efficacy? J Headache Pain 16(Suppl 1):A160

3. Bracaglia M et al (2015) O017. Cortical functional correlates of responsiveness to short-lasting preventive intervention with ketogenic diet (KD) in migraine: a multimodal evoked potentials study. J Headache Pain 16(Suppl 1):A58

4. Porcaro C et al (2015) 0027. Sub-cortical sources of the somatosensory pathway are hypoactive in migraine interictally: a Functional Source Separation analysis. J Headache Pain 16(Suppl 1):A55

5. Coppola G et al (2015) 0025 . Excitability of the motor cortex in migraine changes with the distance from the last attack. J Headache Pain 16(Suppl 1):A158

6. Anastasio MG et al (2015) 0044. Frequency-dependent habituation deficit of the nociceptive blink reflex in migraine with and without aura. J Headache Pain 16(Suppl 1):A59

Correspondence: paolo.martelletti@uniroma1.it

Dipartimento di Medicina Clinica e Molecolare Sapienza, University of Rome, Rome, Italy

(c) The Author(s). 2017 Open Access This article is distributed under the terms of the Creative Commons Attribution 4.0 International License (http://creativecommons.org/licenses/by/4.0/), which permits unrestricted use, distribution, and reproduction in any medium, provided you give appropriate credit to the original author(s) and the source, provide a link to the Creative Commons license, and indicate if changes were made. 